\title{
Solving the Problems for Optimum Thickness of Protective Clothing in a Way of Improvement Based Particle Swarm Optimization
}

\author{
JinYang Zhang ${ }^{1+}$, LiuYang $\mathrm{Xu}^{2}$ and JiaQi Yang ${ }^{3}$ \\ ${ }^{1}$ Wuhan University of Technology
}

\begin{abstract}
This paper is the answer thesis to the questions of the Chinese University Students' Mathematical Modeling Contest (A) in 2018. For the temperature distribution of high-temperature protective clothing, a one-dimensional thermal-conduction equation model established to obtain the numerical solution. And, a multi-objective optimization model is set up for various thicknesses of protective clothing in ideal evaluation method and the approximate optimal solution is obtained in collaborative optimization algorithm of multiple cities. Compared to the classic algorithm, the independently developed algorithm features stronger resistance to local convergence and is easier to obtain a better solution.

For Question 1, the numerical solution is obtained for the one-dimensional thermal-conduction equation in Crank-Nicolson scheme and the temperature distribution of protective clothing is attained. The numerical solution is calculated and obtained for this equation in finite difference method and Crank-Nicolson scheme. About Question 2, based on Question 1, an ideal evaluation method based multi-objective optimization model is set up to obtain the optimum thickness of the Layer II of protective clothing. This paper considers the constrained conditions given in the title as optimization objectives and converts the multi-objective optimization model into single-objective optimization model in ideal evaluation method.

As to Question 3, based on Question 2, this paper finds the solution for the multi-objective optimization problem in collaborative optimization algorithm of multiple cities. Application of this algorithm avoids enormous time consumption caused by traverse strategy. And, the sequent analysis gives an example analyzing the independently developed algorithm better than the other swarm smart algorithms against local convergence.

This paper is advantageously characterized to obtain the numerical solution of a one-dimensional thermalconduction equation in a more accurate finite difference scheme and solves the multi-objective optimization problem in swarm smart algorithm. Compared to the classic algorithm, the independently developed algorithm features stronger resistance to local convergence and is easier to obtain a better solution.
\end{abstract}

Keywords: One-dimensional thermal-conduction equation, Crank-Nicolson scheme, ideal evaluation method, and collaborative optimization algorithm of multiple cities

\section{Recitation of Question}

\subsection{Background}

Under the subject, the given special clothing for work under hot environment is composed of three layers, i.e. Layers I, II, and III. And, Layer I is directly in contact with the external environment, Layer III is separated from the skin by an air layer which is called Layer IV.

A manikin whose body temperature is controlled by $37^{\circ} \mathrm{C}$ is used for experiments. The experimental data information of clothing may be attained by recording the temperature outside the manikin skin

\subsection{Recitation of question}

Question 1: The temperature results outside the manikin skin are measured to calculate the temperature distribution on the layers of protective clothing.

\footnotetext{
+ Corresponding author. Tel.: + 8613719459314; fax: +862034013956 .

E-mail address: zjy_whut@163.com.
} 
Question 2: For work under hot environment, it is required that the optimum thickness of Layer II inside the clothing should be determined according to a specified constrained condition.

Question 3: Based on Question 2, the experimental conditions are changed to determine the optimum thicknesses of Layers II and IV under the given environment.

\section{Analysis of Questions}

\subsection{Idea schematic}

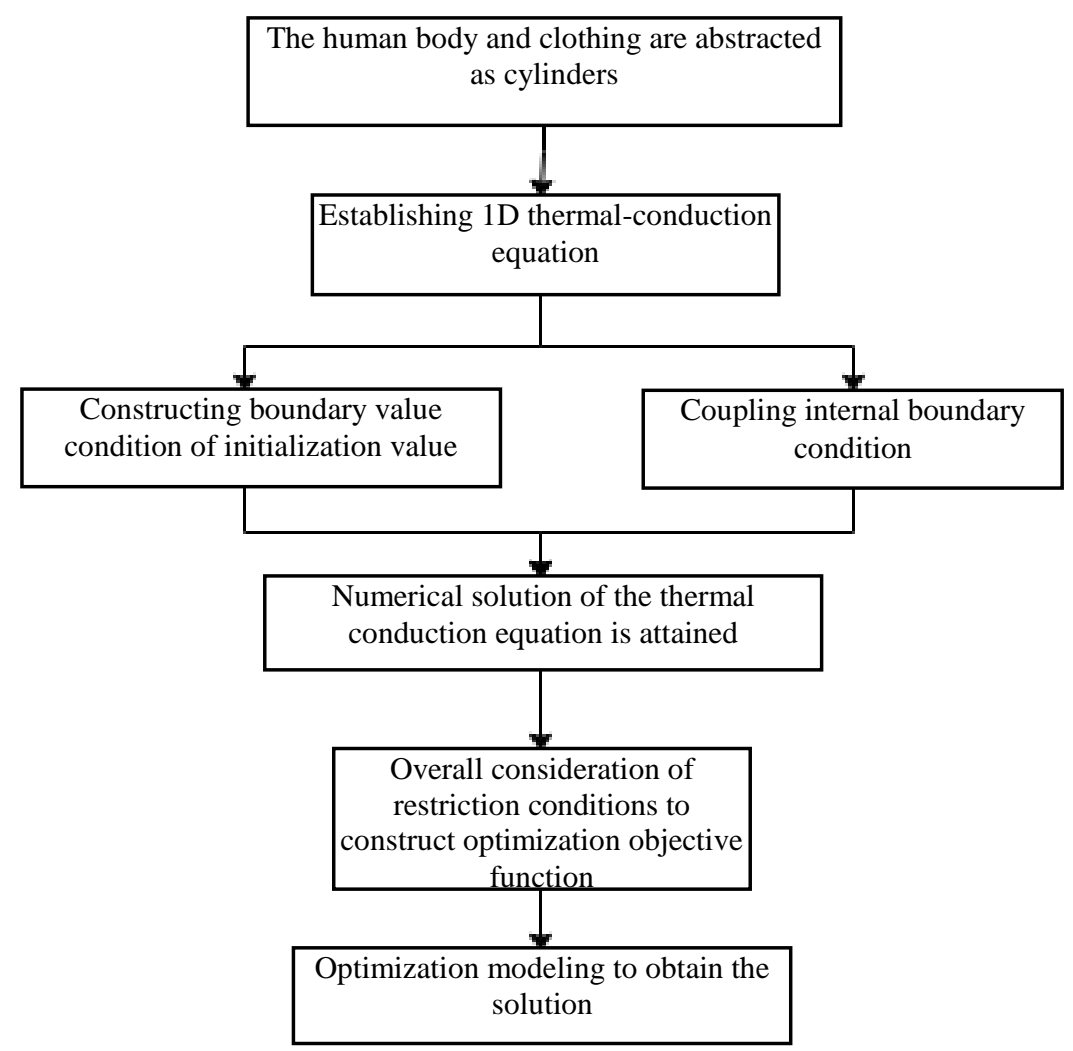

Figure 1: Idea Schematic of Modeling

\subsection{Analysis of questions}

Considering Question 1, this paper describes the temperature distribution of the layers of protective clothing by one-dimensional thermal conduction modeling and finds the solution in finite difference method.

With regard to Question 2, this paper converts it into a problem of multi-objective optimization and finds the solution in an improved swarm smart algorithm.

For Question 3, it is necessary to determine the optimum thicknesses of Layers II and IV under conditions similar to those of Question 2. It is converted into a multi-objective optimization model.

\section{Modeling and Obtaining the Solution of Question 1}

\subsection{Preparations}

The thermal conduction model needs to consider the physical properties of the materials. Relevant properties of the materials may be obtained as shown in the following table:

Table 1: Parameters for Each Layer of Material of Protective Clothing

\begin{tabular}{ccccc}
\hline Layers & Density & Specific & Thermal conductivity & Thickness \\
\hline Layer I & 300 & 1377 & 0.082 & 0.6 \\
Layer II & 862 & 2100 & 0.37 & $0.6-25$ \\
Layer III & 74.2 & 1726 & 0.045 & 3.6 \\
Layer IV & 1.18 & 1005 & 0.028 & $0.6-6.4$ \\
\hline
\end{tabular}


Where, $\alpha_{i}$ is the thermal conductivity coefficient of the material and the taken value of $\alpha_{i}$ is related to the specific heat $C_{i}$, density $\rho_{i}$ and thermal conductivity $k_{i}$, which may be expressed as:

$$
\alpha_{i}=\frac{k_{i}}{C_{i} \cdot \rho_{i}}
$$

\subsection{Modeling of one-dimensional thermal-conduction equations}

The bodies wearing protective clothing are considered as isotropic cylinders that are coaxially nested, as shown in Figure 2. Therefore, the complicated three-dimensional thermal conduction model may be simplified as one-dimensional form, and its numerical solution may be accurately obtained in the CrankNicolson scheme of finite difference method. Figure 3 shows the schematic of a simplified model.

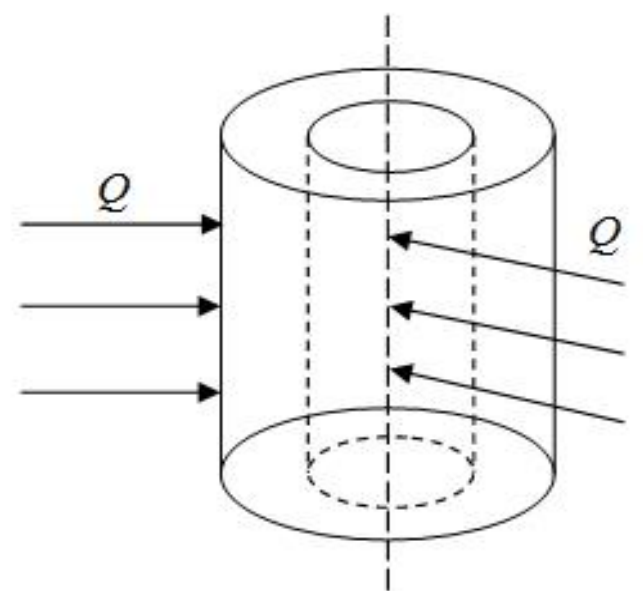

Figure 2: Schematic of a Body Model

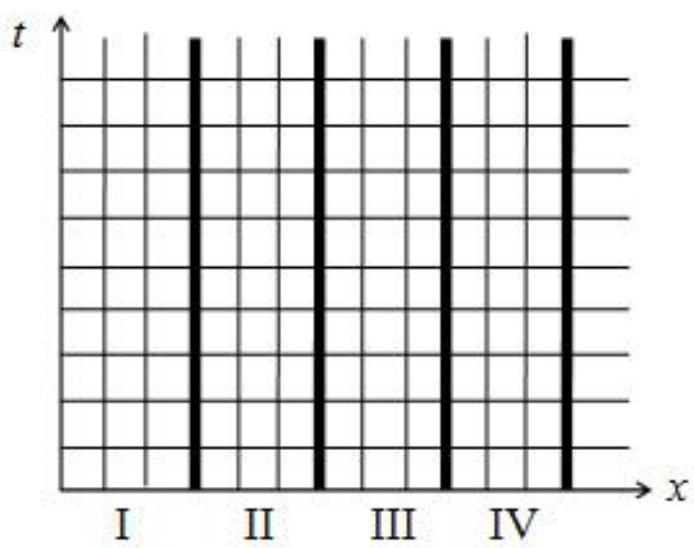

Figure 3: Schematic of a One-dimensional Thermal Conduction Model

According to Literature [1], the heat transmission between the solid materials of protective clothing mainly features thermal conduction and the air layer may possibly have three modes of transmission, i.e. thermal conduction, thermal convection and thermal radiation. And, full study was carried out in Literatures $[2,3]$. According to these literatures, the temperature change of the layers for protective clothing may be described with a thermal conduction model.

The one-dimensional partial differential equation model and expressed as follows:

$$
\left\{\begin{array}{l}
\frac{\partial U}{\partial t}-\alpha_{i} \frac{\partial^{2} U}{\partial x^{2}}=0 \quad i=1, \cdots, 4 \\
U_{i}=U_{i+1} \\
k_{i} \frac{\partial U_{i}}{\partial x}=k_{i+1} \frac{\partial U_{i+1}}{\partial x} \\
0<t<T_{\max } \quad 0<x<L
\end{array}\right.
$$

In above, $i=1, \cdots, 4$ is Layers I, II, III and IV. $\alpha_{i}$ is the thermal conductivity coefficient of the material layers. The second equation restricts that the temperatures at the boundaries between layers are equal. The third equation means the function relationship that the thermal conduction capacity between the material layers should meet. $k_{i}$ is the thermal conductivity of the layer of material.

\subsection{Determining the initial value condition}

At the moment of $t=0$, the temperature of the layers inside the protective clothing is stabilized at fixed value. This paper approximates it to the manikin constant body temperature of $37^{\circ} \mathrm{C}$. Layer I of the protective clothing is directly kept in contact with external hot environment, the temperature will be suddenly changed, of which numerical value directly depends on the external ambient temperature. In Question 1, the given ambient temperature $T_{e}$ is $75^{\circ} \mathrm{C}$. Therefore, the following may be obtained: 


$$
\left\{\begin{array}{l}
U\left(\left.x\right|_{x \neq 0}, 0\right)=37 \\
U(0,0)=T_{e}=75
\end{array}\right.
$$

\subsection{Processing the information of external boundary value}

The ambient temperature $T_{e}$ is considered as the left boundary value condition of the equation and the interpolated skin temperature change function $T_{L}$ as the right boundary value condition, i.e.:

$$
\left\{\begin{array}{l}
U(0, t)=T_{e} \\
U(L, t)=T_{L}
\end{array}\right.
$$

\subsection{Obtained results}

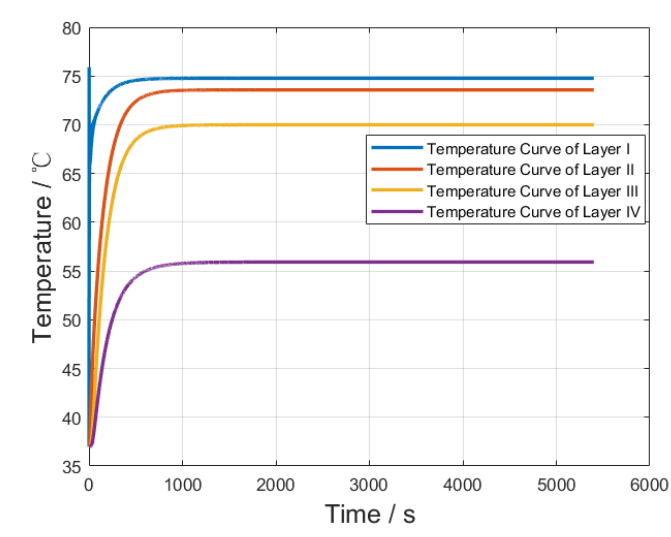

Figure 4: Temperature of Layers vs. Time

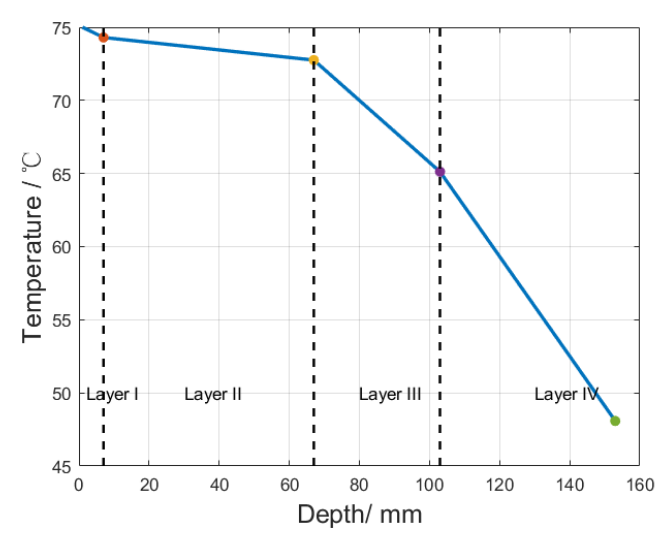

Figure 5: Temperature of Layers Vs Thickness of Protective Clothing

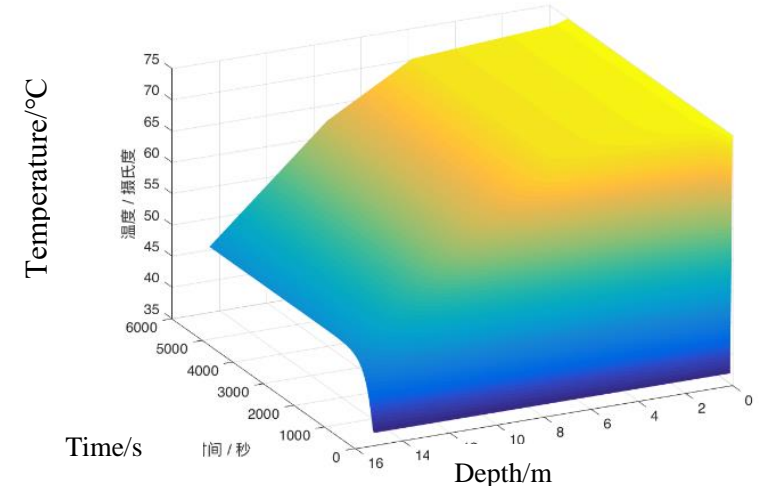

Figure 6: Three-dimensional Image of Temperature Vs Time and Depth Graph for Temperature of Layers Vs Thickness of Protective Clothing

\section{Modeling and Obtaining the Solution of Question 2}

\subsection{Description and analysis of question 2}

Essentially, Question 2 is an optimization problem. This paper thinks that the manikin's constant temperature condition should be used as the new right boundary value condition for obtaining the solution. According to Literatures [4,5], it can be converted into a constant-heat core layer and varying-temperature skin layer as shown in following: 


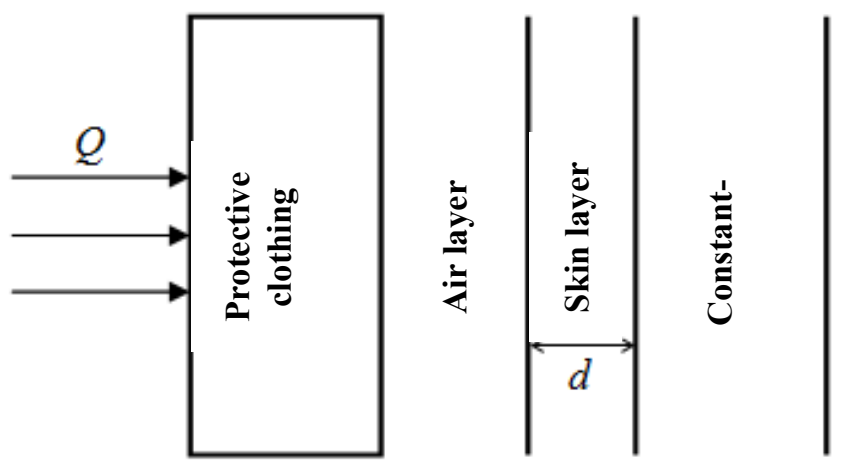

Figure 7: Structural Schematic of a Body Model Wearing Protective Clothing

Different from the model of Question 1, in the above Figure 7, an additional skin layer is provided to establish the one-dimensional thermal conduction model based on Question 1. The right boundary value condition is constructed for obtaining the solution with constant temperature of the manikin's heatgeneration core.

\subsection{Determining the physical coefficient of the skin layer}

In this paper, the thickness of the skin layer is about $3.7 \mathrm{~mm}$, the $\rho_{5}=2300, C_{5}=350$ and $k_{5}=0.35$ are used as the information of thermally physical parameters of Layer $\mathrm{V}$ and the coefficient of thermal conductivity of Layer V, so $\alpha_{5}$ may be obtained. So, the Equations in Model 1 may be changed to:

$$
\left\{\begin{array}{l}
\frac{\partial U}{\partial t}+\alpha_{i} \frac{\partial^{2} U}{\partial x^{2}}=0 \\
U_{i}=U_{i+1} \\
k_{i} \frac{\partial U_{i}}{\partial x}=k_{i+1} \frac{\partial U_{i+1}}{\partial x} \\
i=1, \cdots, 5
\end{array}\right.
$$

\subsection{Ideal evaluation method based thickness optimization modeling}

The heat-insulation effect of the protective clothing will be better with its increased thickness. However, too thick protective clothing may cause additional manufacturing cost and possibly have an adverse influence on the working efficiency. Therefore, this paper thinks that the following three objectives should be considered for solving Question 2.

$$
\left\{\begin{array}{l}
\min f_{1}=l_{2} \\
\min f_{2}=T\left\{\sum_{t} \mathrm{U}(L, t) \geq 44\right\} \\
\min f_{3}=U(L, S)
\end{array}\right.
$$

The first equation means that it is hoped to find the smallest possible thickness of Layer II ; second means that the desired time that the skin external layer exceeds $44^{\circ} \mathrm{C}$ should be as short as possible; third means the temperature of the skin layer at final moment should be as low as possible. Moreover, for these three objectives, the subject gives corresponding constrained conditions:

$$
\left\{\begin{array}{l}
0.6 \leq f_{1} \leq 25 \\
f_{2} \leq 300 \\
f_{3} \leq 47
\end{array}\right.
$$

Because there are not many feasible solutions, it is necessary to consider obtaining the solution of the multi-objective programming model in ideal evaluation method, of which rough idea is explained as follows: 


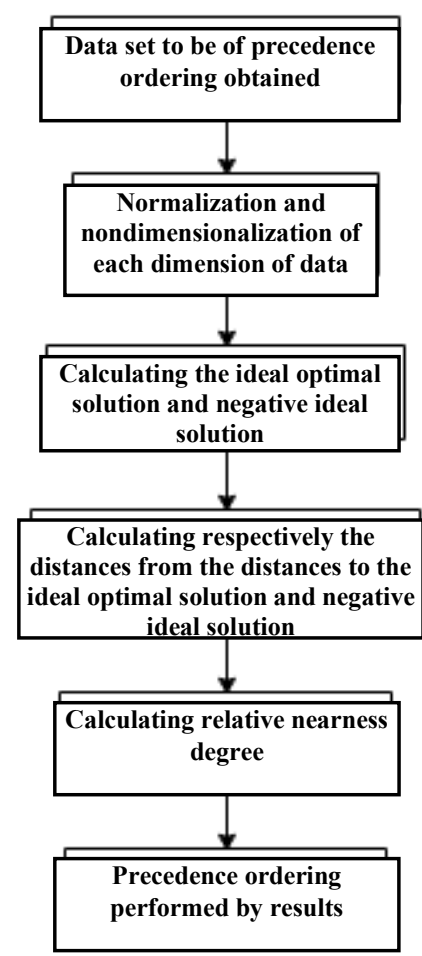

Figure 8: Idea Chart of TOPSIS Evaluation Method

\section{Modeling and Obtaining the Solution of Question 3}

\subsection{Description and analysis of question 3}

Based on Question 2, the thickness of Layer IV is additionally used as the constrained objective in Question 3. Same as Question 2, it is converted into an ideal optimal solution based multi-objective optimization model. And, the solution is obtained in an independently developed collaborative optimization algorithm of multiple cities.

\subsection{New independently developed collaborative optimization algorithm of multiple cities}

The classic particle swarm algorithm features high speed of convergence but is easy to be fallen into partial optimal solution. Literature [6] gives some current prevailing swarm smart algorithms. In general, these algorithms are inspired by the idea of classic particle swarm that uses swarm collaboration to realize artificial intelligence.

This paper uses a new independently developed collaborative optimization algorithm of multiple cities $(\mathrm{COMC})$, which is inspired by the classic particle swarm optimization algorithm. The rough idea is briefly explained as follows: 


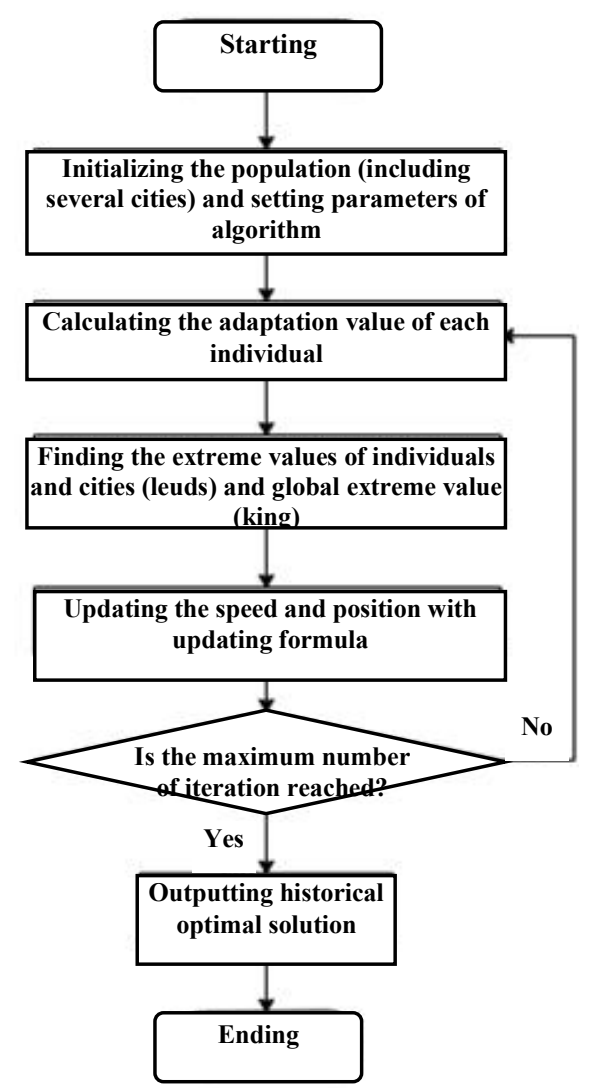

Figure 9: Flow Chart of Collaborative Optimization Algorithm of Multiple Cities

The setting parameters of the above figure may be divided into as follows:

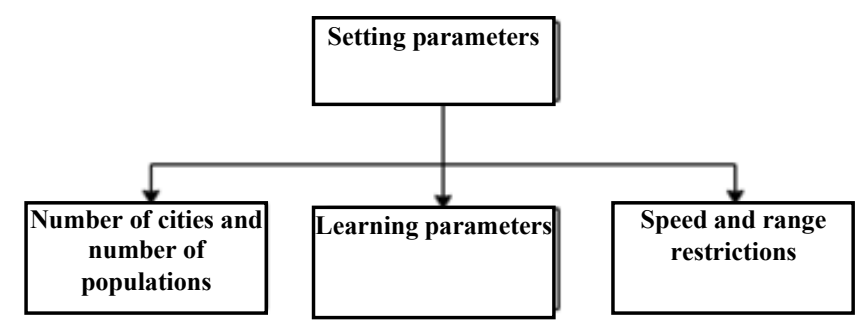

Figure 10: Schematic for Parameters Setting of Collaborative Optimization Algorithm of Multiple Cities

For the first difference between collaborative optimization algorithm of multiple cities and conventional particle swarm algorithm, the former has many cities of collaboration for searching optimal values in the area of obtaining the solution. During each iteration, each city has a leader to lead all the residents in this city to search the optimal value. This leader is called leud leading this city, who is the resident with highest adaptability. There is a king for leuds, who guides the leuds to march for the optimal value. Similar to the leuds, the kings of generations are different but they can represent the best result attained with algorithm of this iteration.

According to the above idea, a hierarchical organization of king-leud-resident may be formed for the information communication of the colonies. In this communication mode, the real colonies' behavior mode is simulated.

With reference to the position updating idea of particle swarm algorithm, this algorithm still updates the speed of each generation to determine the particle position of next generation. For the difference of this algorithm, it has more speed updating modes than the particle swarm, i.e. four modes as shown in the following figure: 


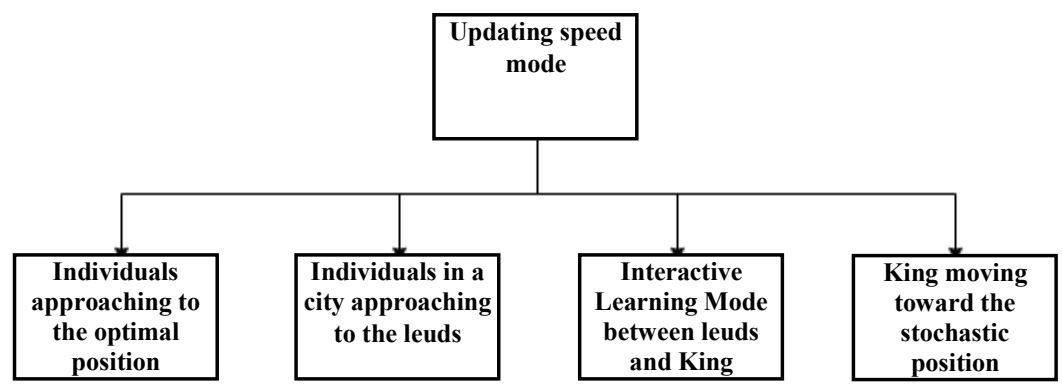

Figure 11: Schematic for Speed Updating Modes of Collaborative Optimization Algorithm of Multiple Cities

Because of strict hierarchical organization, the residents in a city cannot directly approach to the king but may pre-approach to the leud of the city. Instructed by the leud, they explore the adjacent optimal position. In this way, the single optimal-value approximation mode of the classic particle swarm is decomposed into many steps of approximation.

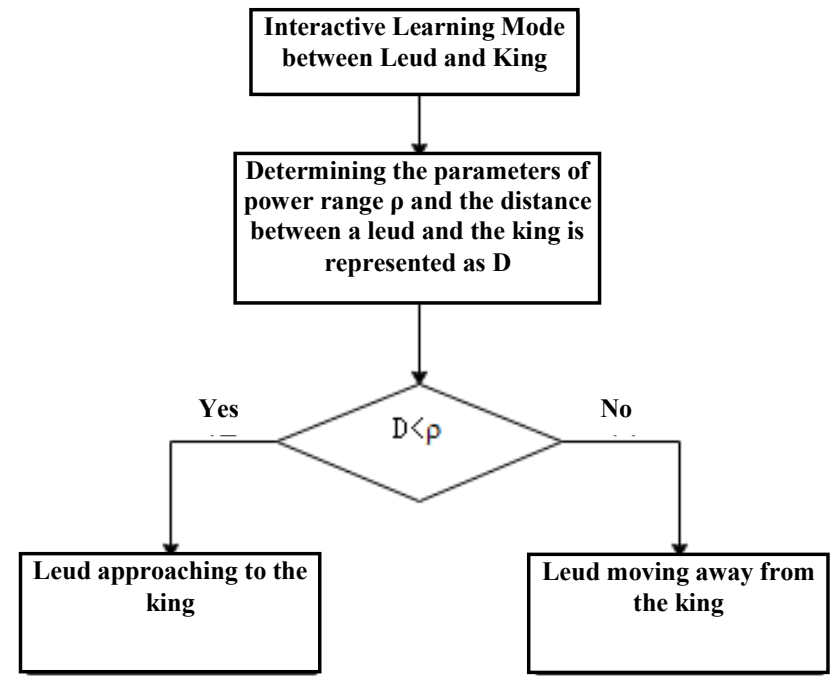

Figure 12: Schematic for Interactive Learning Mode between Leud and King

As shown in the above figure, the interactive strategy between a leud and the king depends on the current distance $D$ between them and the parameters $\rho$ of power range. If the distance between a leud and the king is long, the leud approaches to the king at a speed which is directly proportional to the current distance; if the distance between a leud and the king is short, the leud moves away from the king at a speed which is directly proportional to the current distance. In addition, in order to praise the particle as the king, this particle has more rights to explore its surrounding zone, which are reflected by a new additionally generated random number of the speed updating formula.

\subsection{Obtained results}

The above optimization model is solved in this algorithm with 3 cities, 10 residents and an iteration algebra of 50. The finally attained optimal thickness of Layer II is $l_{2}=22.7 \mathrm{~mm}$ and that of Layer IV is $l_{4}=4.5 \mathrm{~mm}$.

The above two values are substituted into the thermal conduction equation model. By simulation, the temperature change curves at the final moment and on the skin surface are attained and as shown respectively as well as Three-dimensional Graph for Relationship of Three Variables of Optimal Thickness and Convergence Graph in Figures 13 to 16: 


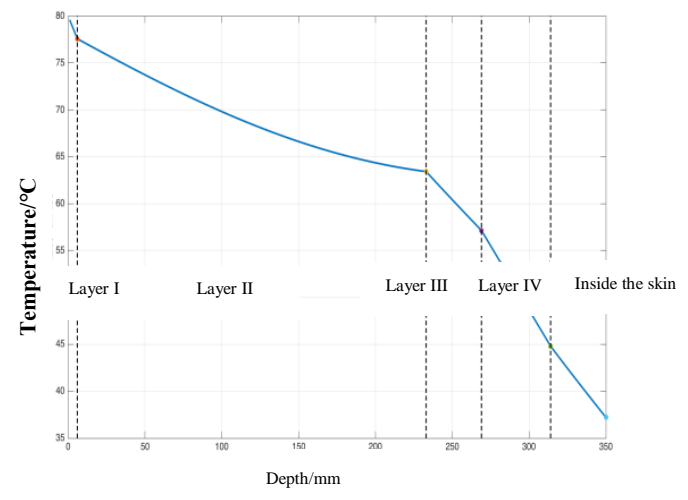

Figure 13: Temperature at Final Moment vs. Depth

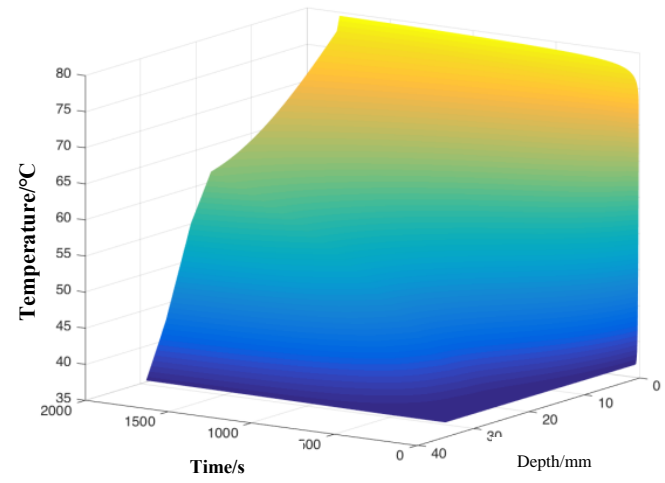

Figure 15: Three-dimension

al Graph

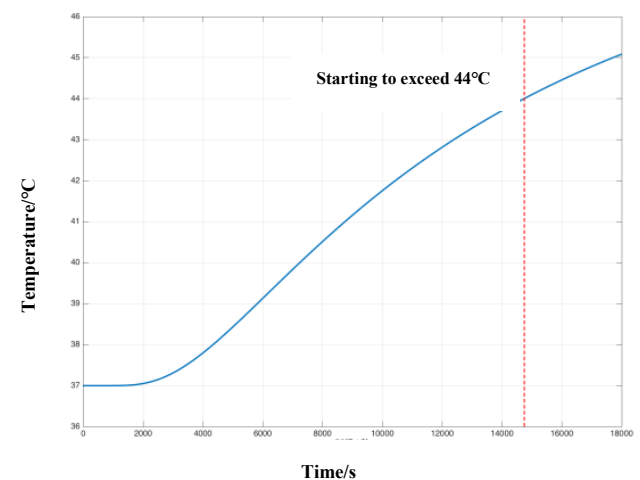

Figure 14: Temperature on Skin Surface vs. Time

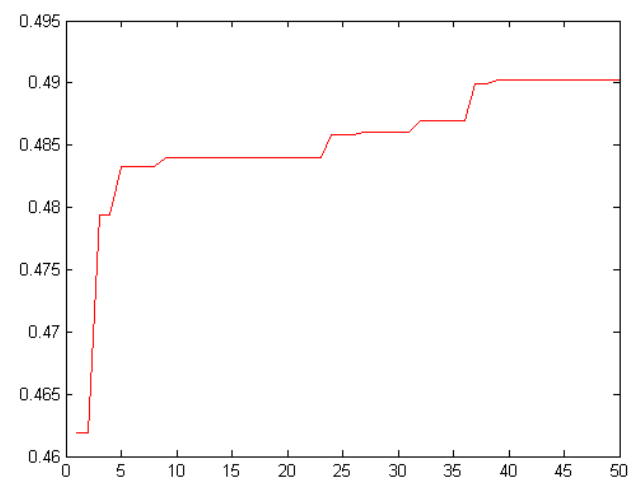

Figure 16: Convergence Graph

\subsection{Algorithm analysis}

Testing is performed with Schaffer function and the testing function is:

$$
y=\frac{0.5+\left(\left(\sin \left(x_{1}^{2}+x_{2}^{2}\right)^{0.5}\right)-0.5\right)}{\left(1+0.001\left(x_{1}^{2}+x_{2}^{2}\right)\right)^{2}}
$$

Table 2: Comparison to Parameters of Three Smart Algorithms

\begin{tabular}{cccc}
\hline & Average Adaptability & $\begin{array}{c}\text { Optimal } \\
\text { Solution }\end{array}$ & $\begin{array}{c}\text { Negative Ideal } \\
\text { Solution }\end{array}$ \\
\hline COMC & 702 & $-1.06 \times 10^{-5}$ & 0.00036 \\
Bat Algorithm & 530 & -0.1285 & 1.7336 \\
Firefly Algorithm & 220 & -0.2714 & 1.7515 \\
\hline
\end{tabular}

Because the minimum value of this function is attained at $(0,0)$, the collaborative optimization algorithm of multiple cities used in this paper is the most approximate and better than the other two smart algorithms according to the data as shown in Table 2.

\section{References}

[1] Y. Su, Y. Wang, J. Li. Research Progress of Heat Transfer Mechanism of Air Gap under Firefighter Protective Clothing. Journal of Textile Research, 2016 (01):167-172.

[2] D. Torvi, J. Dale, B. Faulkener. Influence of Air Gaps On Bench-Top Test Results of Flame Resistant Fabrics. Journal of Fire Protection Engineering, 1999,10 (1):1-12.

[3] D. Torvi. Heat Transfer In Thin Fibrous Materials Under High Heat Flux Condition. Edmonton University of Alberta, 1997. 
[4] X. Luo. System Design and Optimization of Thermal Manikin. Donghua University,2016.

[5] G,Havenith. Heat balance when wearing protective clothing. Ann Occup Hyg, 1999,43 (5):289-296

[6] S. Lin, C. Dong, M. Chen, et al. Overview of New Swarm Smart Optimization algorithm. Computer Engineering and Applications, 2018 (12):1-9. 